\title{
Effects of MHD on the Unsteady Thin Flow of Non-Newtonian Oldroyd -B Fluid over an Oscillating Inclined Belt Through a Porous Medium
}

\author{
Sundos Bader, Ahmed M, Abdulhadi \\ Department of Mathematic, College of Science ,University of Baghdad, Baghdad, Iraq \\ Email:badersundos@gmail.com and ahm6161@yahoo.com
}

\begin{abstract}
The unsteady (MHD) thin film flow of an incompressible Oldroyd -B fluid over an oscillating inclined belt making a certain angle with the horizontal through a porous mediumis analyzed .The analytical solution of velocity field is obtained by a semi-numerical technique optimal homotopy asymptotic method $(O H A M)$. Finally the influence of various dimensionless parameters emerging in the model on velocity field is analyzed by graphical illustrations.
\end{abstract}

\section{Introduction}

In everyday life and in engineering flow of non-Newtonian fluid are frequently occurred .It is ubiquitous in nature and technologies. Therefore to understand there mechanics is essential in most application .Most of it problems appeared in several examples are polymer solution ,paints ,certain oils, exocitic lubricants ,colloidal and suspension solutions, clay coatings and cosmetic products .In non-Newtonian fluids Thin film flows have a large varying of practical applications in nonlinear science and engineering industries .

For modeling of non-Newtonian Fluid flow problem we used several model like (second grade, third grade , Maxwell fluid ,Oldroyd-B ) ..ect fluid model by which we got linear or nonlinear ordinary differential equation or partial differential equations which may be solved exactly, analytically or numerically. For solution of these problems different varieties of methods are used, in which (ADM,VAM,HAM,HPM,OHAM) are frequently used. In this work we have modeled a partial differential equations by using oldroyd-B fluid model. Solution of the problem is obtained by using OHAM. Fetecau et al. [3] studied the exact solutions of an Oldroyd-B fluid over a flat plate on constantly accelerating flow. In the following year, Fetecau et al. [4] obtained the exact solutions of the transient oscillating motion of an Oldroyd-B fluids in cylindrical domains. Haitao and Mingyu [5] investigated the series solution of an Oldroyd-B fluid by using the sine and Laplace transformations for the plane Poiseuille flowand plane couette flow. Lie L. [7] studied the effect of MHD flow for an Oldroyd-B fluid between two oscillating cylinders .Khan et al. Burdujan [2] discussed the solution for the flow of an incompressible Oldroyd-B fluid between two cylinders . Shahid et al. [12] studied the solution of the steady and unsteady flow of Oldroyd-B fluid by using Laplace and Fourier series. Shah et al. [13] studied the solution by using OHAM of thin film flow of third grade fluid on moving inclined plane. Marinca et al. [11] studied the approximate solution of non-linear steady flow of fourth grade fluid by using OHAM. Marinca et al [10] discussed the approximate solution of the unsteady viscous flow over a shrinking cylinder by using OHAM method . Kashkari [6] studied the OHAM solution of nonlinear Kawahara equation. For comparison HPM, VHPM and VIM method is used but OHAM ismore successful method. . Anakira et al. [1] the analytical solution of delay differential equation by using OHAM. Mabood et al. [8,9] investigated the approximate solution of non-linear Riccati differential equation by using OHAM . Taza Gul [14] studied the unsteady magnetohydrodynamics (MHD) thin film flow of an incompressible Oldroyd-B fluid over an oscillating inclined belt making a certain angle with the horizontal.

In this paper, we study the unsteady (MHD) thin film flow of an incompressible Oldroyd -B fluid over an oscillating inclined belt making a certain angle with the horizontal in porous medium . the solution of velocity field is obtained by using a semi-numerical technique optimal homotopy asymptotic method (OHAM). Finally, we analyzed the influence of various dimensionless parameters emerging in the model on velocity field is analyzed by graphical illustrations.

\section{Governing Equation}

The continuity and momentum equations for an unsteady magnetic hydrodynamic (MHD) incompressible flow over an inclined belt through porous medium, defined by the following equations

$$
\begin{aligned}
& \operatorname{div} V=0 \\
& \rho \frac{D V}{D t}=\operatorname{div} \boldsymbol{T}+\rho g \sin \theta+\boldsymbol{J} \times \boldsymbol{B}+R
\end{aligned}
$$

Where $T$ denoted the Cauchy stress, $V$ is the velocity vector of fluid, $\rho$ is the fluid density , $g$ is the external body force, $\boldsymbol{B}$ is the magnetic field, and $\boldsymbol{J}$ is current density (or conduction current).

The Cauchy stress tensor $T$ for incompressible Oldroyd -B viscous fluid is defined by the constitutive equation

DOI: $10.9790 / 5728-1204052840 \quad$ www.iosrjournals.org $28 \mid$ Page




$$
T=-p I+\boldsymbol{S}
$$

where $p$ is the pressure, I the identity tensor, the extra stress tensor $\boldsymbol{S}$ satisfies

$$
\boldsymbol{S}+\lambda_{1} \frac{D \boldsymbol{S}}{D t}=\mu\left(1+\lambda_{2} \frac{D}{D t}\right) A
$$

Where $\mu$ the dynamic viscosity, $\lambda_{1}, \lambda_{2}$ are the relaxation and retardation times respectively, and $A$ is the first Rivlin-Ericksen tensor and $\frac{D}{D t}$ the upper convected derivative defined as follows.

$$
\begin{aligned}
& A=L+L^{T}, \quad L=\operatorname{grad} V \\
& \frac{D \boldsymbol{S}}{D t}=\frac{\partial \boldsymbol{S}}{\partial t}+V \cdot \nabla \boldsymbol{S}-L \boldsymbol{S}-\boldsymbol{S} L^{T} \\
& \frac{D \boldsymbol{A}}{D t}=\frac{\partial \boldsymbol{A}}{\partial t}+V \cdot \nabla \boldsymbol{A}-L \boldsymbol{A}-\boldsymbol{A} L^{T}
\end{aligned}
$$

Where $\nabla$ is the gradient operator .

We assume that the velocity field and the shear stress of the form

$$
V=(u(y, t), 0,0) \text { and } \boldsymbol{S}=\boldsymbol{S}(y, t)
$$

Where $u(y, t)$ is the velocity. Since $V$ is dependent of $y$ and $t$, we also assume that $\boldsymbol{S}$ dependent only on $y$ and $t$

If the fluid being at rest up to the moment $\mathrm{t}=0$, then

We can get

$$
\boldsymbol{S}(y, 0)=0, y>0
$$

$$
\begin{aligned}
& S_{x x}+\lambda_{1}\left(\frac{\partial S_{x x}}{\partial t}-2 \frac{\partial u}{\partial y} S_{y x}\right)=-2 \mu \lambda_{2}\left(\frac{\partial u}{\partial y}\right)^{2} \\
& S_{x y}+\lambda_{1}\left(\frac{\partial S_{x y}}{\partial t}-\frac{\partial u}{\partial y} S_{y y}\right)=\mu \frac{\partial u}{\partial y}+\mu \lambda_{2} \frac{\partial^{2} u}{\partial t \partial y} \\
& S_{y y}+\lambda_{1} \frac{\partial S_{y y}}{\partial t}=0
\end{aligned}
$$

then Eq.(12) reduces to

$$
S_{y y}=C(y) e^{-\frac{t}{\lambda_{1}}}
$$

where $C(y)$ is arbitrary function . From Eq.(9), $C(y)=0$.

where $S_{x z}=S_{y z}=S_{z z}=0, S_{x y}=S_{y x}$.

\section{Statement of the problem}

This paper consider a thin film flow of a non-Newtonian Oldroyd -B fluid on an oscillating inclined belt .We assumed the flow is unsteady, laminar, incompressible, and the pressure gradient is zero . The force of gravity has been initiated the motion of a layer of liquid in the downward direction .Thickness, $\delta$, of the liquid layer is assumed to be uniform. A magnetic field is applied to the belt in the direction perpendicular to fluid motion, as shown in Fig. (1). The boundary conditions are given by

$$
\begin{aligned}
& u(0, t)=V \cos \omega t, t>0 \\
& \frac{\partial u(y, t)}{\partial y}=0 \text { as } y \rightarrow \infty, t>0
\end{aligned}
$$

where $\omega$ is oscillating belt

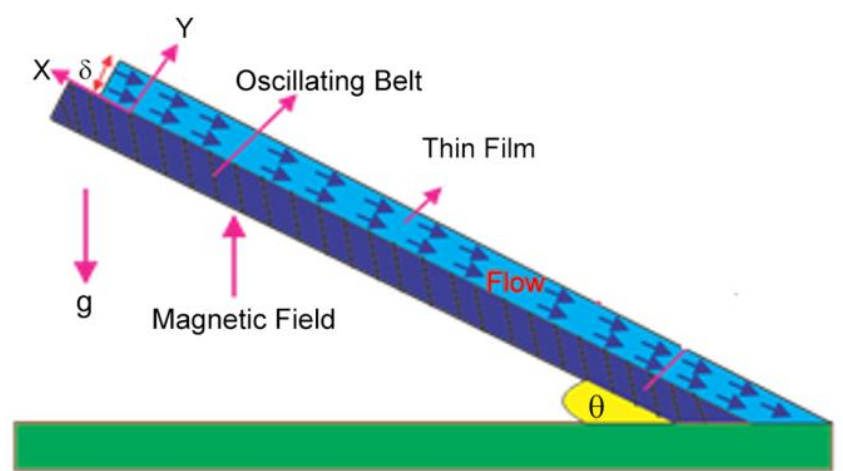

Fig.(1): Geometry of the problem

IV. Momentum and continuity Equation

By using the above argument we will write the formula of the momentum equations which governing the magnetohydrodynamic in $x$-direction as follows: 


$$
\rho \frac{\partial u}{\partial t}=-\frac{\partial p}{\partial x}+\frac{\partial S_{x y}}{\partial y}+\rho g \sin \theta-\sigma B_{0}^{2} u-\frac{\mu}{K} u
$$

Differential Eq.(11) with respect to y, we get

$$
\begin{aligned}
& \frac{\partial S_{x y}}{\partial y}+\lambda_{1} \frac{\partial}{\partial y}\left(\frac{\partial S_{x y}}{\partial t}-\frac{\partial v}{\partial y} S_{y y}\right)=\mu \frac{\partial^{2} u}{\partial y^{2}}+\mu \lambda_{2} \frac{\partial^{3} u}{\partial t \partial y^{2}} \\
& \left(1+\lambda_{1} \frac{\partial}{\partial t}\right) \frac{\partial S_{x y}}{\partial y}-\lambda_{1}\left(\frac{\partial^{2} u}{\partial y^{2}} S_{y y}+\frac{\partial v}{\partial y} \frac{S_{y y}}{\partial y}\right)=\mu\left(1+\lambda_{2} \frac{\partial}{\partial t}\right) \frac{\partial^{2} u}{\partial y^{2}}
\end{aligned}
$$

Multiply Eq.(18) by $\left(1+\lambda_{1} \frac{\partial}{\partial t}\right)^{-1}$,we get

$$
\frac{\partial S_{x y}}{\partial y}=\frac{\lambda_{1}}{\left(1+\lambda_{1} \frac{\partial}{\partial t}\right)}\left(\frac{\partial^{2} u}{\partial y^{2}} S_{y y}+\frac{\partial v}{\partial y} \frac{S_{y y}}{\partial y}\right)+\mu \frac{\left(1+\lambda_{2} \frac{\partial}{\partial t}\right)}{\left(1+\lambda_{1} \frac{\partial}{\partial t}\right)} \frac{\partial^{2} u}{\partial y^{2}}
$$

Substitute Eq.(19) into Eq.(16),we get

$$
\begin{gathered}
\rho \frac{\partial u}{\partial t}=-\frac{\partial p}{\partial x}+\left(\frac{\lambda_{1}}{\left(1+\lambda_{1} \frac{\partial}{\partial t}\right)}\left(\frac{\partial^{2} u}{\partial y^{2}} S_{y y}+\frac{\partial u}{\partial y} \frac{S_{y y}}{\partial y}\right)+\mu \frac{\left(1+\lambda_{2} \frac{\partial}{\partial t}\right)}{\left(1+\lambda_{1} \frac{\partial}{\partial t}\right)} \frac{\partial^{2} u}{\partial y^{2}}\right)+\rho g \sin \theta-\sigma B_{0}^{2} u \\
-\frac{\mu}{K} u
\end{gathered}
$$

Multiply Eq.(20) by $\left(1+\lambda_{1} \frac{\partial}{\partial t}\right)$, we obtain

$$
\begin{gathered}
\rho\left(1+\lambda_{1} \frac{\partial}{\partial t}\right) \frac{\partial u}{\partial t}=-\left(1+\lambda_{1} \frac{\partial}{\partial t}\right) \frac{\partial p}{\partial x}+\left(\lambda_{1}\left(\frac{\partial^{2} u}{\partial y^{2}} S_{y y}+\frac{\partial v}{\partial y} \frac{S_{y y}}{\partial y}\right)+\mu\left(1+\lambda_{2} \frac{\partial}{\partial t}\right)\right. \\
\left.\frac{\partial^{2} u}{\partial y^{2}}\right)+\rho g \sin \theta-\left(1+\lambda_{1} \frac{\partial}{\partial t}\right) \sigma B_{0}^{2} u-\left(1+\lambda_{1} \frac{\partial}{\partial t}\right) \frac{\mu}{K} u
\end{gathered}
$$

Since from Eqs.(13) and (9), then $S_{y y}$ is reduced to zero, which demonstrates that $C(y)=0$.Therefore from Eqs.(13) and (19) and in the presence of zero pressure gradient, we get

$$
\begin{gathered}
\rho\left(1+\lambda_{1} \frac{\partial}{\partial t}\right) \frac{\partial u}{\partial t}=\mu\left(1+\lambda_{2} \frac{\partial}{\partial t}\right) \frac{\partial^{2} u}{\partial y^{2}}+\rho g \sin \theta-\left(1+\lambda_{1} \frac{\partial}{\partial t}\right) \sigma B_{0}^{2} u \\
-\left(1+\lambda_{1} \frac{\partial}{\partial t}\right) \frac{\mu}{K} u
\end{gathered}
$$

Divide the above equation by $\rho$, we get

$$
\begin{gathered}
\left(1+\lambda_{1} \frac{\partial}{\partial t}\right) \frac{\partial u}{\partial t}=\frac{\mu}{\rho}\left(1+\lambda_{2} \frac{\partial}{\partial t}\right) \frac{\partial^{2} u}{\partial y^{2}}+\rho g \sin \theta-\left(1+\lambda_{1} \frac{\partial}{\partial t}\right) \frac{\sigma B_{0}^{2}}{\rho} u- \\
\frac{1}{\rho} \frac{\mu}{K}\left(1+\lambda_{1} \frac{\partial}{\partial t}\right) u
\end{gathered}
$$

Introducing non-dimensional variables

$$
\begin{gathered}
u=\frac{\bar{u}}{V}, y=\frac{\bar{y}}{\delta}, t=\frac{\mu \bar{t}}{\rho \delta^{2}}, k_{1}=\frac{\lambda_{1} \mu}{\rho \delta^{2}}, k_{2}=\frac{\lambda_{2} \mu}{\rho \delta^{2}}, \omega=\frac{\rho \bar{\omega} \delta^{2}}{\mu}, m=\frac{\delta^{2} \rho g \sin \theta}{\mu V}, \\
M=\frac{\sigma B_{0}^{2} \delta^{2}}{\mu},
\end{gathered}
$$

Where $\omega$ is the oscillating parameter, $k_{1}$ is the relaxation parameter, $k_{2}$ is the retardation parameter,$m$ is the gravitational parameter and $\mathrm{M}$ is the Magnetic parameter.

We find Eqs.(24), (14) and (15) in dimensionless forms(for simplicity the mark " - " neglected)

and

$$
\left(1+k_{1} \frac{\partial}{\partial t}\right) \frac{\partial u}{\partial t}=\left(1+k_{2} \frac{\partial}{\partial t}\right) \frac{\partial^{2} u}{\partial y^{2}}+m-M\left(1+k_{1} \frac{\partial}{\partial t}\right) u-\frac{\delta^{2}}{K}\left(1+k_{1} \frac{\partial}{\partial t}\right) u
$$

$$
u(0, t)=\cos (\omega t) \text { and } \frac{\partial u(1, t)}{\partial y}=0
$$

\section{Basic ideas of Optimal Homotopy Asymptotic Method}

To illustrate the basic ideas of optimal homotopy asymptotic method, we will consider the following general of partial differential equation of the form

$$
A(u(y, t))+Q(y, t)=0 \quad B\left(u(y, t), \frac{\partial u(y, t)}{\partial y}\right)=0 \quad y \in \Omega
$$

Where $A$ is a differential operator, $B$ is a boundary operator, $u(y, t)$ is unknown function, $\Omega$ is the problem domain and $Q(y, t)$ is known analytic function. The operator $A$ can be decomposed as 


$$
A=L+N
$$

Where $L$ is linear operator and $N$ is a nonlinear operator . According to OHAM , one can construct an optimal homotopy $\varphi(y, t, p): \Omega \times[0,1] \rightarrow R$ which satisfies

$$
H(\varphi(y, t, p), p)=(1-p)\left[L(\varphi(y, t, p)+Q(y, t)]-H\left(p, c_{i}\right)[A(\varphi(y, t, p)+Q(y, t)]=0\right.
$$

Where $p \in[0,1]$ is an embedding parameter and $H\left(p, c_{i}\right)$ is non -zero auxiliary function, which can be define in the form

$$
H\left(p, c_{i}\right)=p c_{1}+p^{2} c_{2}+p^{3} c_{3}+\cdots
$$

Where $c_{1}, c_{2}, c_{3}, \ldots$ are called convergence controle parameters and will be determined accordingly to obtain an approximate solution, $\varphi(y, t, p)$ is expanded in series about $p$ as

$$
\varphi\left(y, t, p, c_{1}, c_{2}, c_{3} \ldots\right)=u_{0}(y, t)+\sum_{k \geq 1} u_{k}\left(y, t, p, c_{1}, c_{2}, c_{3} \ldots\right) p^{k}
$$

If $p=0$, then $H\left(0, c_{i}\right)=0, \varphi(y, t, 0)=u_{0}(y, t)$ and from Eq.(29), we get

and

$$
H(\varphi(y, t, p), p)=\left[L\left(u_{0}(y, t)\right)+Q u_{0}(y, t)\right]=0
$$

If $p=1$, then $H\left(1, c_{i}\right)=0, \varphi(y, t, 1)=u(y, t)$ and from Eq.(29), we get

$$
H(\varphi(y, t, 1), 1)=H\left(1, c_{i}\right)[A(u(y, t))+Q u(y, t)]
$$

Now by inserting Eq.(31) into Eq.(27)and equating the coefficient of like powers of $p$, we obtain the zero,first and second order are given by

$$
\begin{gathered}
L\left(u_{0}(y, t)\right)+Q(y, t)=0 \quad B\left(u_{0}(y, t), \frac{\partial u_{0}(y, t)}{\partial y}\right)=0 \\
L\left(u_{1}(y, t)\right)=c_{1} N_{0}\left(u_{0}(y, t)\right) \quad B\left(u_{1}(y, t), \frac{\partial u_{1}(y, t)}{\partial y}\right)=0 \\
L\left(u_{2}(y, t)\right)-L\left(u_{1}(y, t)\right)=c_{2} N_{0}\left(u_{0}(y, t)\right)+c_{1}\left[L\left(u_{1}(y, t)\right)+N_{1}\left(u_{0}(y, t), u_{1}(y, t)\right)\right] \\
B\left(u_{2}(y, t), \frac{\partial u_{2}(y, t)}{\partial y}\right)=0
\end{gathered}
$$

And hence , the general governing equation for $u_{j}(y, t)$ are given by

$$
\begin{aligned}
& L\left(u_{j}(y, t)\right)-L\left(u_{j-1}(y, t)\right) \\
& =c_{j} N_{0}\left(u_{0}(y, t)\right)+\sum_{i=1}^{j-1} c_{i}\left[L\left(u_{j-1}(y, t)\right)+N_{j-1}\left(u_{0}(y, t), u_{1}(y, t), \ldots, u_{j-1}(y, t)\right)\right] \\
& \quad B\left(u_{j}(y, t), \frac{\partial u_{j}(y, t)}{\partial y}\right)=0, j=2,3, \ldots \ldots
\end{aligned}
$$

Where $N_{m}\left(u_{0}(y, t), u_{1}(y, t), \ldots, u_{m}(y, t)\right)$ is the coefficient of $p^{m}$, obtained by expanding $N\left(\varphi\left(y, t, p, c_{1}, c_{2}, c_{3} \ldots\right)\right)$ is series with respect to the embedding parameter $p$.

$$
N\left(\varphi\left(y, t, p, c_{1}, c_{2}, c_{3} \ldots\right)\right)=N_{0}\left(u_{0}(y, t)\right)+\sum_{j=1}^{\infty} N_{j}\left(u_{0}(y, t), u_{1}(y, t), \ldots, u_{j}(y, t)\right) p^{j}
$$

The convergence of the series in $\mathrm{Eq}(39)$ depends upon the auxiliary constants $c_{1}, c_{2}, c_{3}, \ldots$ if it converges at $p=1$, then the mth order approximation $u$ is

$$
u\left(y, c_{1}, c_{2}, c_{3} \ldots\right)=u_{0}(y, t)+\sum_{j=1}^{m} u_{j}\left(y, c_{i}\right), i=1,2, \ldots, m
$$

Substituting Eq.(38) into Eq.(27), we get the following expression for the residual error

$$
R\left(y, t, c_{i}\right)=L\left(u\left(y, t, c_{i}\right)\right)+Q(y, t)+N\left(u\left(y, t, c_{i}\right)\right), i=1,2, \ldots, m
$$

If $R\left(y, t, c_{i}\right)=0$, then $u\left(y, c_{1}, c_{2}, c_{3} \ldots\right)$ is the exact solution. To find the optimal value of

$$
J\left(c_{i}\right)=\int_{a}^{b} R^{2}\left(y, t, c_{i}\right) d y
$$

Where the value $\mathrm{a}$ and $\mathrm{b}$ depend on the given problem . the unknown convergence control parameters $c_{i}(i=$ $1,2, \ldots, m)$ can be optimally identified from the conditions

$$
\frac{\partial J}{\partial c_{i}}=0 \quad i=1,2, \ldots \ldots, m
$$

Where the constants $c_{1}, c_{2}, c_{3} \ldots$ can be determined by using Numerical methods (least square method, Ritz\method, Galerkin's method and collocation method ect.). 
Rewrite the Eq.(33) in the form

\section{Solution of the problem}

$$
\frac{\partial^{2} u}{\partial y^{2}}+k_{2} \frac{\partial}{\partial t}\left(\frac{\partial^{2} u}{\partial y^{2}}\right)-\left(M+\frac{\delta^{2}}{K}\right) u-\left(\left(M+\frac{\delta^{2}}{K}\right) k_{1}+1\right) \frac{\partial u}{\partial t}-k_{1}\left(\frac{\partial^{2} u}{\partial t^{2}}\right)+m=0
$$

and the boundary conditions are

$$
u(0, t)=\cos (\omega t) \text { and } \frac{\partial u(1, t)}{\partial y}=0
$$

we choose linear operator

$$
\begin{gathered}
L(u(y, t))=\frac{\partial^{2} u}{\partial y^{2}}+m \\
A(u(y, t))=\frac{\partial^{2} u}{\partial y^{2}}+k_{2} \frac{\partial}{\partial t}\left(\frac{\partial^{2} u}{\partial y^{2}}\right)-\left(M+\frac{\delta^{2}}{K}\right) u-\left(\left(M+\frac{\delta^{2}}{K}\right) k_{1}+1\right) \frac{\partial u}{\partial t}-k_{1}\left(\frac{\partial^{2} u}{\partial t^{2}}\right) \\
+m
\end{gathered}
$$

and

$$
Q(y, t)=0
$$

substitute Eqs.(45) and (46) into Eq.(29), we get

$$
\begin{gathered}
(1-p)\left[\frac{\partial^{2} u}{\partial y^{2}}+m\right]-H\left(p, c_{i}\right)\left[\frac{\partial^{2} u}{\partial y^{2}}+k_{2} \frac{\partial}{\partial t}\left(\frac{\partial^{2} u}{\partial y^{2}}\right)-\left(M+\frac{\delta^{2}}{K}\right) u-\left(\left(M+\frac{\delta^{2}}{K}\right) k_{1}+1\right)\right. \\
\left.\frac{\partial u}{\partial t}-k_{1}\left(\frac{\partial^{2} u}{\partial t^{2}}\right)+m\right]=0
\end{gathered}
$$

substitute Eq.(30) and (31) into Eq.(48), we obtain

Zero-order problem given by

$$
p^{0}: \frac{\partial^{2} u_{0}}{\partial y^{2}}=-m
$$

and the boundary conditions are

$$
u_{0}(0, t)=\cos (\omega t) \text { and } \frac{\partial u_{0}(1, t)}{\partial y}=0
$$

The solution of Eq.(49) given by

$$
u_{0}(y, t)=-\frac{m}{2} y^{2}+m y+\operatorname{Cos}(\omega t)
$$

First -order problem given by

$$
\begin{aligned}
p^{1}: \frac{\partial^{2} u_{1}}{\partial y^{2}}=(1 & \left.+c_{1}\right) \frac{\partial^{2} u_{0}}{\partial y^{2}}+\left(1+c_{1}\right) m+c_{1} k_{2} \frac{\partial}{\partial t}\left(\frac{\partial^{2} u_{0}}{\partial y^{2}}\right)-c_{1}\left(M+\frac{\delta^{2}}{K}\right) u_{0} \\
- & c_{1}\left(\left(M+\frac{\delta^{2}}{K}\right) k_{1}+1\right) \frac{\partial u_{0}}{\partial t}-c_{1} k_{1}\left(\frac{\partial^{2} u_{0}}{\partial t^{2}}\right)
\end{aligned}
$$

and the boundary conditions are

The solution of Eqs.(52)and (53) is given by

$$
u_{1}(0, t)=\cos (\omega t) \text { and } \frac{\partial u_{1}(1, t)}{\partial y}=0
$$




$$
\begin{aligned}
& u_{1}(y, t)=\cos (\omega \mathrm{t})-\frac{1}{6} m M y^{3} c_{1}+\frac{1}{24} m M y^{4} c_{1}-\frac{m y^{3} \delta^{2} c_{1}}{6 K}+\frac{m y^{4} \delta^{2} c_{1}}{24 K}-\frac{1}{2} M y^{2} \cos (\omega \mathrm{t}) c_{1} \\
&-\frac{y^{2} \delta^{2} \cos (\omega \mathrm{t}) c_{1}}{2 K}+\frac{1}{2} y^{2} \omega \sin (\omega \mathrm{t}) c_{1}+\frac{1}{3} m M c_{1}^{2}+\frac{m \delta^{2}}{3 K}+M \cos (\omega \mathrm{t}) c_{1}^{2} \\
&+\frac{\delta^{2} \cos (\omega \mathrm{t}) c_{1}^{2}}{K}-\omega \sin (\omega \mathrm{t}) c_{1}^{2}+\frac{1}{2} y^{2} \omega^{2} \cos (\omega \mathrm{t}) c_{1} k_{1}+\frac{1}{2} M y^{2} \omega \sin (\omega \mathrm{t}) c_{1} k_{1} \\
&+\frac{y^{2} \delta^{2} \omega \sin (\omega \mathrm{t}) c_{1} k_{1}}{2 K}-\omega^{2} \cos (\omega \mathrm{t}) c_{1}^{2} k_{1}-M \omega \sin (\omega \mathrm{t}) c_{1}^{2} k_{1} \\
&-\frac{\delta^{2} \omega \sin (\omega \mathrm{t}) c_{1}^{2} k_{1}}{K}
\end{aligned}
$$

Second -order problem given by

$$
\begin{gathered}
p^{2}: \frac{\partial^{2} u_{2}}{\partial y^{2}}=\left(1+c_{1}\right) \frac{\partial^{2} u_{1}}{\partial y^{2}}+c_{1} k_{2} \frac{\partial}{\partial t}\left(\frac{\partial^{2} u_{1}}{\partial y^{2}}\right)-c_{1}\left(M+\frac{\delta^{2}}{K}\right) u_{1}-c_{1}\left(\left(M+\frac{\delta^{2}}{K}\right) k_{1}+1\right) \\
\frac{\partial u_{1}}{\partial t}-c_{1} k_{1}\left(\frac{\partial^{2} u_{1}}{\partial t^{2}}\right)+c_{2} \frac{\partial^{2} u_{0}}{\partial y^{2}}+c_{2} k_{2} \frac{\partial}{\partial t}\left(\frac{\partial^{2} u_{0}}{\partial y^{2}}\right)-c_{2}\left(M+\frac{\delta^{2}}{K}\right) u_{0} \\
-c_{2}\left(M+\frac{\delta^{2}}{K}\right) k_{1} \frac{\partial u_{0}}{\partial t}+c_{2} m-c_{2} \frac{\partial u_{0}}{\partial t}-c_{2} k_{1}\left(\frac{\partial^{2} u_{0}}{\partial t^{2}}\right)
\end{gathered}
$$

and the boundary conditions are

$$
u_{2}(0, t)=\cos (\omega t) \text { and } \frac{\partial \mathrm{u}_{2}(1, \mathrm{t})}{\partial \mathrm{y}}=0
$$

The solution of Eqs.(55) and (56) is given by

$$
\begin{aligned}
u_{2}(\mathrm{y}, \mathrm{t})=\cos (\omega \mathrm{t} & -\frac{1}{2} y^{2}\left(M+\frac{\delta^{2}}{K}\right) \cos (\omega \mathrm{t}) c_{1}+\left(\frac{m y^{4}}{18}-\frac{m y^{6}}{720}+\frac{1}{6} y^{3} \cos (\omega \mathrm{t})\right) c_{1}^{2}-\frac{1}{2} y^{2}\left(M+\frac{\delta^{2}}{K}\right)^{2}\left(\frac{m}{3}\right. \\
& +\cos (\omega \mathrm{t})) c_{1}^{3}+\left(M+\frac{\delta^{2}}{K}\right)\left(\frac{m y^{3}}{6}-\frac{m y^{4}}{24}+\frac{1}{2} y^{2} \cos (\omega \mathrm{t})\right) c_{1}\left(1+c_{1}\right)-\frac{1}{6} y^{3}\left(M+\frac{\delta^{2}}{K}\right) \omega c_{2} \\
& -\frac{1}{2} y^{2}\left(M+\frac{\delta^{2}}{K}\right) \cos (\omega \mathrm{t}) c_{2}+\frac{1}{2} y^{2} \omega \sin (\omega \mathrm{t}) c_{2}+\frac{1}{24} m y^{4}\left(M+\frac{\delta^{2}}{K}\right) c_{2}^{2} \\
& +\frac{1}{2} y^{2} \omega^{2} \cos (\omega \mathrm{t}) c_{1} k_{1}+\frac{1}{2} y^{2}\left(M+\frac{\delta^{2}}{K}\right) \omega^{2} \cos (\omega \mathrm{t}) c_{1}^{2} k_{1}-\frac{1}{24} y^{4}\left(M+\frac{\delta^{2}}{K}\right) \omega^{2} \cos (\omega \mathrm{t}) c_{1}^{2} k_{1} \\
& +\frac{1}{2} y^{2}\left(M+\frac{\delta^{2}}{K}\right) \omega^{2} \cos (\omega \mathrm{t}) c_{1}^{3} k_{1}-\frac{1}{24} y^{4}\left(M+\frac{\delta^{2}}{K}\right) \omega^{2} \cos (\omega \mathrm{t}) c_{1}^{3} k_{1}+y \cos (\omega \mathrm{t}) c_{1}(1 \\
& \left.+c_{1}\right) k_{1}+\frac{1}{2} y^{2} \omega^{2} \cos (\omega \mathrm{t}) c_{2} k_{1}+\omega \sin (\omega \mathrm{t}) c_{1}^{2}\left(1+\left(M+\frac{\delta^{2}}{K}\right) k_{1}\right)+ \\
+ & \frac{1}{2} y^{2}\left(M+\frac{\delta^{2}}{K}\right) \omega \sin (\omega \mathrm{t}) c_{1}^{3}\left(1+\left(M+\frac{\delta^{2}}{K}\right) k_{1}\right)+\frac{1}{2} y^{2} \omega \sin (\omega \mathrm{t})\left(1+c_{1}\right)(1+(M \\
& \left.\left.+\frac{\delta^{2}}{K}\right) k_{1}\right)+\frac{1}{2} y^{2} \omega^{2} \cos (\omega \mathrm{t}) c_{1}^{2}\left(1+c_{1}\right)\left(1+\left(M+\frac{\delta^{2}}{k}\right) k_{1}\right)-\frac{1}{2} y^{2} \omega^{3} \sin (\omega \mathrm{t}) c_{1}^{2} k_{1}(1+(M \\
& \left.\left.+\frac{\delta^{2}}{K}\right) k_{1}\right)+\frac{1}{24} y^{4} \omega^{3} \sin (\omega \mathrm{t}) c_{1}^{2} k_{1}\left(1+\left(M+\frac{\delta^{2}}{K}\right) k_{1}\right)+\frac{1}{24} y^{4} \omega^{3} \sin (\omega \mathrm{t})^{2} c_{1}^{2} k_{1}(1+(M \\
& \left.\left.+\frac{\delta^{2}}{K}\right) k_{1}\right) \\
& -\frac{1}{24} y^{4}\left(M+\frac{\delta^{2}}{K} \omega \sin (\omega \mathrm{t}) c_{1}^{2}\left(1+\left(M+\frac{\delta^{2}}{K}\right) k_{1}\right)\right. \\
& \left.-\frac{1}{24} y^{4}\left(M+\frac{\delta^{2}}{K}\right) \omega \sin (\omega \mathrm{t})^{2} c_{1}^{2}\left(1+\left(M+\frac{\delta^{2}}{K}\right) k_{1}\right)\right)-\frac{1}{2} y^{2} \omega^{3} \sin (\omega \mathrm{t}) c_{1}^{3} k_{1}^{3}(1+(M \\
& \left.\left.+\frac{\delta^{2}}{K}\right) k_{1}\right)+\mathrm{Q} \\
& (57)
\end{aligned}
$$

Where $\mathrm{Q}$ is a function of $\omega, t, \delta^{2}, M, m, y, k_{1}, c_{1}, c_{2}, K$ and $k_{2}$.

Substituting Eqs.(51),(54) and(57) into Eq.(39), we get

$u(y, t)=u_{0}(\mathrm{y}, \mathrm{t})+u_{1}(\mathrm{y}, \mathrm{t})+u_{2}(\mathrm{y}, \mathrm{t})$ 


$$
\begin{aligned}
& u(y, t)=-\frac{m}{2} y^{2}+m y+\cos (\omega t)+\cos (\omega t)-\frac{1}{6} m M y^{3} c_{1}+\frac{1}{24} m M y^{4} c_{1}-\frac{m y^{3} \delta^{2} c_{1}}{6 K}+\frac{m y^{4} \delta^{2} c_{1}}{24 K} \\
& -\frac{1}{2} M y^{2} \cos (\omega \mathrm{t}) c_{1}-\frac{y^{2} \delta^{2} \cos (\omega \mathrm{t}) c_{1}}{2 K}+\frac{1}{2} y^{2} \omega \sin (\omega \mathrm{t}) c_{1}+\frac{1}{3} m M c_{1}^{2}+\frac{m \delta^{2} c_{1}^{2}}{3 K} \\
& +M \cos (\omega \mathrm{t}) c_{1}^{2}+\frac{\delta^{2} \cos (\omega \mathrm{t}) c_{1}^{2}}{K}-\omega \sin (\omega \mathrm{t}) c_{1}^{2}+\frac{1}{2} y^{2} \omega^{2} \cos (\omega \mathrm{t}) c_{1} k_{1} \\
& +\frac{1}{2} M y^{2} \omega \sin (\omega \mathrm{t}) c_{1} k_{1}+\frac{y^{2} \delta^{2} \omega \sin (\omega \mathrm{t}) c_{1} k_{1}}{2 K}-\omega^{2} \cos (\omega \mathrm{t}) c_{1}^{2} k_{1}-M \omega \sin (\omega \mathrm{t}) c_{1}^{2} k_{1} \\
& -\frac{\delta^{2} \omega \sin (\omega \mathrm{t}) c_{1}^{2} k_{1}}{K}+\cos (\omega \mathrm{t})-\frac{1}{2} y^{2}\left(M+\frac{\delta^{2}}{K}\right) \cos (\omega \mathrm{t}) c_{1}+\left(\frac{m y^{4}}{18}-\frac{m y^{6}}{720}\right. \\
& \left.+\frac{1}{6} y^{3} \cos (\omega \mathrm{t})\right) c_{1}^{2}-\frac{1}{2} y^{2}\left(M+\frac{\delta^{2}}{K}\right)^{2}\left(\frac{m}{3}+\cos (\omega \mathrm{t})\right) c_{1}^{3}+\left(M+\frac{\delta^{2} \varphi}{K}\right)\left(\frac{m y^{3}}{6}-\frac{m y^{4}}{24}\right. \\
& \left.+\frac{1}{2} y^{2} \cos (\omega \mathrm{t})\right) c_{1}\left(1+c_{1}\right)-\frac{1}{6} y^{3}\left(M+\frac{\delta^{2}}{K}\right) \omega c_{2}-\frac{1}{2} y^{2}\left(M+\frac{\delta^{2}}{K}\right) \cos (\omega \mathrm{t}) c_{2} \\
& +\frac{1}{2} y^{2} \omega \sin (\omega \mathrm{t}) c_{2}+\frac{1}{24} m y^{4}\left(M+\frac{\delta^{2}}{K}\right) c_{2}^{2}+\frac{1}{2} y^{2} \omega^{2} \cos (\omega \mathrm{t}) c_{1} k_{1}+\frac{1}{2} y^{2}(M \\
& \left.+\frac{\delta^{2}}{K}\right) \omega^{2} \cos (\omega \mathrm{t}) c_{1}^{2} k_{1}-\frac{1}{24} y^{4}\left(M+\frac{\delta^{2}}{K}\right) \omega^{2} \cos (\omega \mathrm{t}) c_{1}^{2} k_{1}+\frac{1}{2} y^{2}(M \\
& \left.+\frac{\delta^{2}}{K}\right) \omega^{2} \cos (\omega \mathrm{t}) c_{1}^{3} k_{1}-\frac{1}{24} y^{4}\left(M+\frac{\delta^{2}}{K}\right) \omega^{2} \cos (\omega \mathrm{t}) c_{1}^{3} k_{1}+y \cos (\omega \mathrm{t}) c_{1}\left(1+c_{1}\right) k_{1} \\
& +\frac{1}{2} y^{2} \omega^{2} \cos (\omega \mathrm{t}) c_{2} k_{1}+\frac{1}{2} y^{2}\left(M+\frac{\delta^{2}}{K}\right) \omega \sin (\omega \mathrm{t}) c_{2} k_{1} \\
& +\frac{1}{2} y^{2}\left(M+\frac{\delta^{2}}{K}\right) \omega \sin (\omega \mathrm{t}) c_{1}^{3}\left(1+\left(M+\frac{\delta^{2}}{K}\right) k_{1}\right)+\frac{1}{2} y^{2} \omega \sin (\omega \mathrm{t})\left(1+c_{1}\right)(1+(M \\
& \left.\left.+\frac{\delta^{2}}{K}\right) k_{1}\right)+\frac{1}{2} y^{2} \omega^{2} \cos (\omega \mathrm{t}) c_{1}^{2}\left(1+c_{1}\right)\left(1+\left(M+\frac{\delta^{2}}{K}\right) k_{1}\right)-\frac{1}{2} y^{2} \omega^{3} \sin (\omega \mathrm{t}) c_{1}^{2} k_{1}(1+(M \\
& \left.\left.+\frac{\delta^{2}}{K}\right) k_{1}\right)+\frac{1}{24} y^{4} \omega^{3} \sin (\omega \mathrm{t}) c_{1}^{2} k_{1}\left(1+\left(M+\frac{\delta^{2}}{K}\right) k_{1}\right)+\frac{1}{24} y^{4} \omega^{3} \sin (\omega \mathrm{t})^{2} c_{1}^{2} k_{1}(1+(M \\
& \left.\left.+\frac{\delta^{2}}{K}\right) k_{1}\right)+\frac{1}{2} y^{2}\left(M+\frac{\delta^{2}}{K}\right) \omega \sin (\omega \mathrm{t}) c_{1}^{2}\left(1+\left(M+\frac{\delta^{2}}{K}\right) k_{1}\right) \\
& -\frac{1}{24} y^{4}\left(M+\frac{\delta^{2}}{K} \omega \sin (\omega \mathrm{t}) c_{1}^{2}\left(1+\left(M+\frac{\delta^{2}}{K}\right) k_{1}\right)\right. \\
& \left.-\frac{1}{24} y^{4}\left(M+\frac{\delta^{2}}{K}\right) \omega \sin (\omega \mathrm{t})^{2} c_{1}^{2}\left(1+\left(M+\frac{\delta^{2}}{K}\right) k_{1}\right)\right)-\frac{1}{2} y^{2} \omega^{3} \sin (\omega \mathrm{t}) c_{1}^{3} k_{1}^{3}(1+(M \\
& \left.\left.+\frac{\delta^{2}}{K}\right) k_{1}\right)+\mathrm{Q}
\end{aligned}
$$

substituting the approximate solution of Eq.(59) into Eq.(40) yields the residual and the functional $J$ ,respectively

$J\left(c_{1}, c_{2}\right)=\int_{0}^{1} R^{2}\left(y, t, c_{1}, c_{2}\right) d y$

$$
\begin{aligned}
R\left(y, t, c_{1}, c_{2}\right)=\frac{\partial^{2} u}{\partial y^{2}}+ & k_{2} \frac{\partial}{\partial t}\left(\frac{\partial^{2} u}{\partial y^{2}}\right)-\left(M+\frac{\delta^{2}}{k}\right) u-\left(\left(M+\frac{\delta^{2}}{K}\right) k_{1}+1\right) \frac{\partial u}{\partial t} \\
& -k_{1}\left(\frac{\partial^{2} u}{\partial t^{2}}\right)+m
\end{aligned}
$$

Differentiating Eq.(61) with respect to $c_{1}$ and $c_{2}$, respectively and solving the result Equations to calculation the unknown auxiliary constants $c_{1}$ and $c_{2}$, in the particular cases

$m=0.2, w=0.2, k_{1}=0.5, k_{2}=0.3, M=0.3, t=5, \delta=0.1$ and $K=1$, we obtain

The values of $c_{i}$ for the velocity components

\begin{tabular}{|c|c|}
\hline$c_{1}$ & $c_{2}$ \\
\hline-0.191837933 & -1.688934901 \\
\hline-0.136536081 & 0.804774143 \\
\hline-0.2098634205 & 1.453685377 \\
\hline-0.162677767 & 1.900789022 \\
\hline-0.164379301 & 3.312570207 \\
\hline
\end{tabular}

Table 1: the values of $c_{i}$ for the velocity component 


\section{Numerical results and conclusions}

This section presents the effects of controlling parameters on the velocity profile in the form of graphical and tabulated results .In order to validate the accuracy of our approximate solution via OHAM, we have been used a semi-numerical technique optimal homotopy asymptotic method (OHAM), for solving unsteady (MHD) thin film flow of an incompressible Oldroyd -B fluid over an oscillating inclined belt making a certain angle with the horizontal in porous medium .The effects of pertinent parameters (define fluid behavior and flow geometries) such as gravitational parameter $(\mathrm{m})$, magnetic parameter $(\mathrm{M})$, relaxation time parameter $\left(k_{1}\right)$, retardation time parameter $\left(k_{2}\right)$, permeability parameter $(K)$, the oscillating parameter $\omega$, time $\mathrm{t}$, and the parameters $\left(c_{1}, c_{2}, \delta, y\right)$.All the graphs are plotted by using MATHEMATICA Software .

In table (1), we calculated the values of $c_{i}$ by using least square method .

Fig.(1) shows the physical configuration of the problem . Fig. (2) is depicted to show the changes of the velocity with gravitational parameter $(\mathrm{m})$, it is observed that as $(\mathrm{m})$ increasing then the velocity is decreasing. Fig. (3) the velocity $u$ is plotted for different value of magnetic parameter $M \quad$.The results indicated that the velocity of $u$ is increased with increase magnetic parameter $M$, when $0 \leq \omega<0.37$ but it has the same value when $\omega=0.37$ and the velocity is decreased when $0.37<\omega<0.8$ and it has the same value when $\omega=0.8$ but it is increased when $\omega>2$. Fig.(4) is sketch to show the profiles of the velocity field for different values of the relaxation time parameter $k_{1}$. From this figures, it is obvious to note that the velocity is has the same value with increase the relaxation time parameter when $0 \leq \omega<0.42$ but it is increasing with increase the relaxation time parameter when $0.42<\omega<0.99$ and is has the same value with increase the relaxation time parameter when $\omega=0.42$ but it is increased when $\omega>1.0$.Fig.(5) shows the behavior of the velocity profiles for different values of retardation time parameter $k_{2}$.It is observed that the velocity is increased with increasing the value of retardation time parameter when $0<\omega<0.632$ and it has the same value with increase the relaxation time parameter when $\omega=0.632$ but it is decreasing with increase the relaxation time parameter when $0.632<\omega<1.253$ and it has the same value with increase the relaxation time parameter when $\omega=1.253$.Fig.(6) illustrate the influence of the parameter $\left(c_{2}\right)$ on the velocity field, we can see the velocity is decreasing with increase $c_{2}$ when $0<\omega<0.232$ then the velocity is increasing when $0.232<\omega<0.65$ but it has decreased when increase the value of $\left(c_{2}\right)$ when $0.65<\omega<1.29$ and then it has decreased with increase the value of $c_{2}$ when $\omega>1.29$. Fig. (7) is established to show the behavior of different time $t$, we can see the velocity u is decreasing with increase the time $t$ when $0 \leq \omega<0.7$ but it is increasing with increase the time $t$ when $0.7 \leq \omega<1.387$.Fig.(8) displays the impact of the parameter $c_{1}$ on the velocity field .It can be seen that, the velocity become similar with increasing the value of $c_{1}$.Fig.(9) sketches to show the profiles of the velocity field for different values of the permeability parameter $(K)$.It is observed that the velocity is decreasing with increase the value of the permeability parameter $(K)$ when $0<\omega<0.39$ and the velocity is increasing with increase the value of the permeability parameter $(K)$ on the interval $0.39<\omega<0.82$ but it has decreased with increase the parameter when $\omega>0.82$.Fig.(10) shows the effect of the different values of the parameter $y$ on the velocity .It is seen that the velocity increase as the parameter $y$ increasing with $\omega<0.323$ but it has decreasing with increase the parameter $y$ in the interval $0.323<\omega<0.6$ and then the velocity is increasing when $0.6<\omega<1.35$ but it is increased when $\omega>1.35$ . Figs.(11and 12 ) show the behavior of the velocity for different values gravitational parameter $(\mathrm{m})$ and magnetic parameter(M) .It is seen that the velocity increase as the parameters increasing .Fig(13) indicate that ,by increasing the relaxation time parameter $\left(k_{1}\right)$, the velocity decrease with increase relaxation time parameter. Fig.(14) displays the impact of retardation time parameter $\left(k_{2}\right)$ on the velocity .It seen that the velocity increase with increase retardation time .Figs.(15 and 16) .It is observed that the velocity redues with increasing magnitude the parameter $c_{2}$ and time $t$.Fig.(17) show the behavior of $c_{1}$ on the velocity field, we can seen the velocity has the same value with increase $c_{1}$.Figs.(18 and 19) illustrate the influence of permeability parameter $(K)$ and the oscillating parameter $\omega$. That implies that the velocity reduces with increase the value of permeability parameter $(K)$ and the oscillating parameter $\omega$.

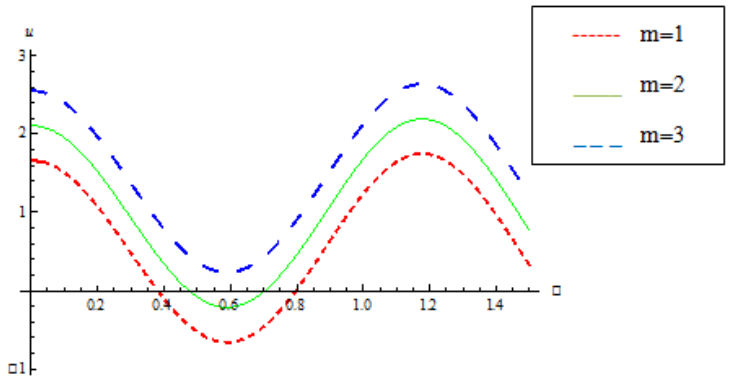

Fig.2:- the velocity u , Eq.(59) for different value of $m$ when keeping other parameters fixed $\{\mathrm{y}=0.5, \delta=0.1$, $\left.\mathrm{c}_{1}=-0.191837933, \mathrm{c}_{2}=-1.688934901, \mathrm{k}_{1}=0.2, \mathrm{k}_{2}=0.3, \mathrm{M}=0.3, \mathrm{t}=5, K=1\right\}$ 


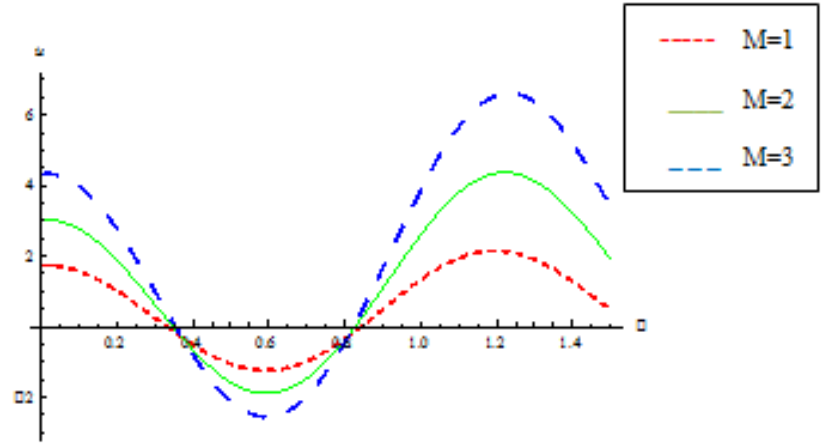

Fig.3:- the velocity u, Eq.( 59) for different value of $\mathrm{M}$ when keeping other parameters fixed $\{\mathrm{y}=0.5, \delta=0.1$, $\left.\mathrm{c}_{1}=-0.191837933, \mathrm{c}_{2}=-1.688934901, \mathrm{k}_{1}=0.2, \mathrm{k}_{2}=0.3, \mathrm{~m}=0.2, \mathrm{t}=5, K=1\right\}$

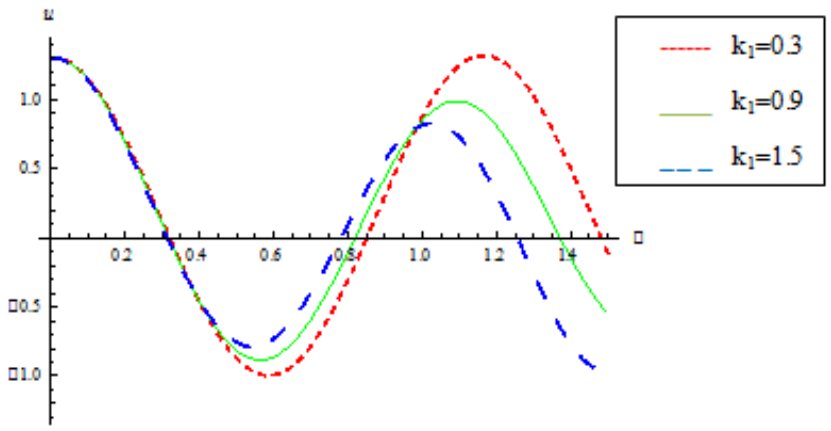

Fig.4:- :- the velocity u , Eq. (59) for different value of $\mathrm{k}_{1}$ when keeping other parameters fixed $\{\mathrm{y}=0.5, \delta=0.1$ , $\left.\mathrm{c}_{1}=-0.191837933, \mathrm{c}_{2}=-1.688934901, \mathrm{M}=0.3, \mathrm{k}_{2}=0.3, \mathrm{~m}=0.2, \mathrm{t}=5, K=1\right\}$

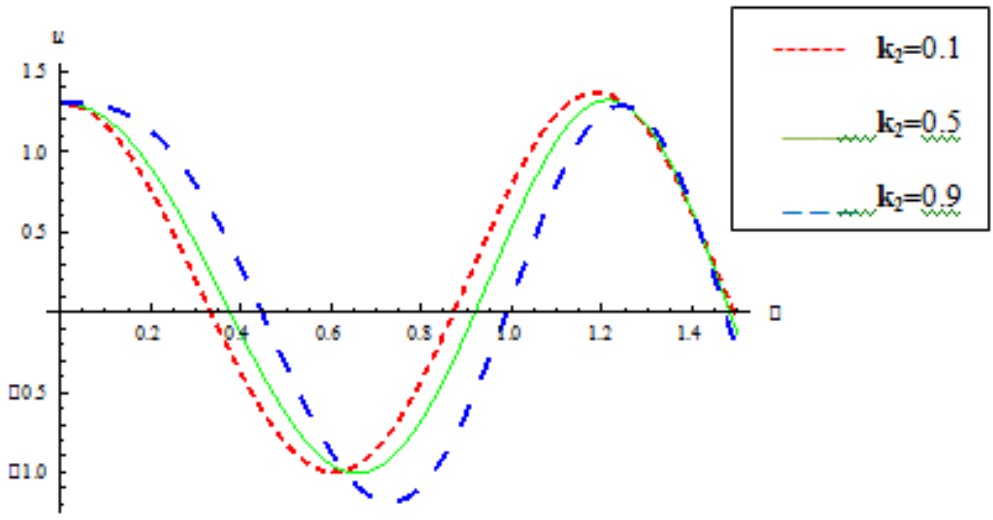

Fig.5:- the velocity u , Eq.( 59) for different value of $\mathrm{k}_{2}$ when keeping other parameters fixed $\{\mathrm{y}=0.5, \delta=0.1$, $\left.\mathrm{c}_{1}=-0.191837933, \mathrm{c}_{2}=-1.688934901, \mathrm{M}=0.3, \mathrm{k}_{1}=0.2, \mathrm{~m}=0.2, \mathrm{t}=5, K=1\right\}$

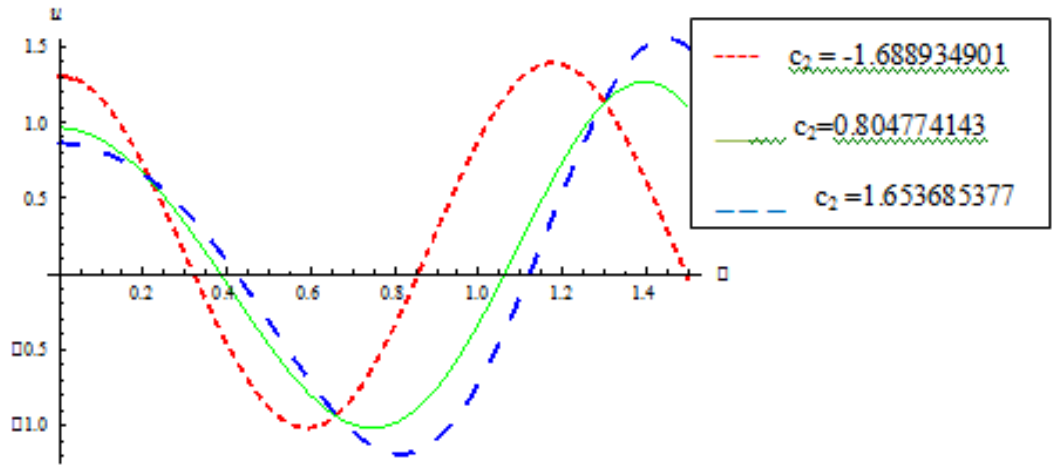

Fig.6:- the velocity u , Eq.( 59) for different value of $c_{2}$ when keeping other parameters fixed $\left\{\mathrm{y}=0.5, \delta=0.1, \mathrm{c}_{1}=-0.191837933, \mathrm{M}=0.3, \mathrm{k}_{1}=0.2, \mathrm{~m}=0.2, \mathrm{k}_{2}=0.3, \mathrm{t}=5, K=1\right\}$ 


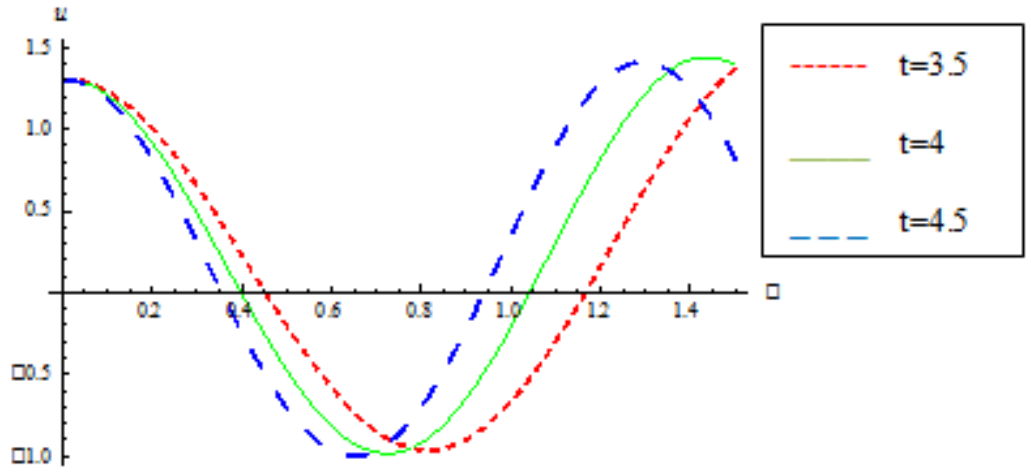

Fig.7 :- the velocity u , Eq.( 59) for different value of $\mathrm{t}$ when keeping other parameters fixed $\left\{\mathrm{y}=0.5, \delta=0.1, \mathrm{c}_{1}=-0.191837933, \mathrm{c}_{2}=-1.688934901, \mathrm{M}=0.3, \mathrm{k}_{1}=0.2, \mathrm{~m}=0.2, \mathrm{k}_{2}=0.3, K=1\right\}$

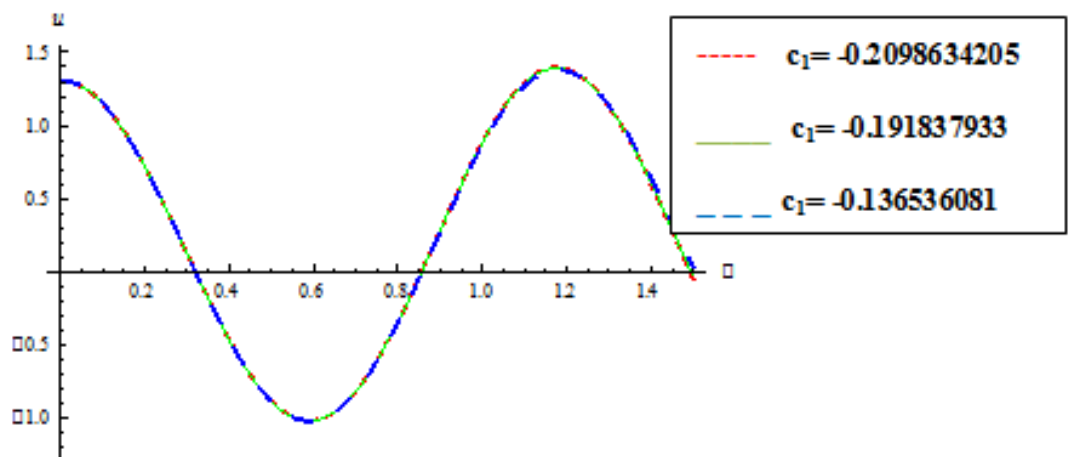

Fig.8 :- the velocity u , Eq. (59) for different value of $c_{1}$ when keeping other parameters fixed $\left\{\mathrm{y}=0.5, \delta=0.1, \mathrm{c}_{2}=-1.688934901, \mathrm{M}=0.3, \mathrm{k}_{1}=0.2, \mathrm{~m}=0.2, \mathrm{t}=5, \mathrm{k}_{2}=0.3, K=1\right\}$

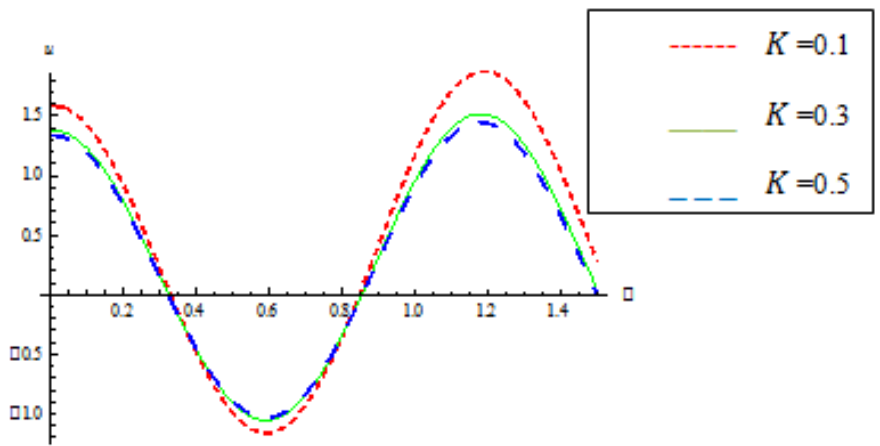

Fig.9:- the velocity u , Eq.( 59) for different value of $K$ when keeping other parameters fixed $\left\{\mathrm{y}=0.5, \delta=0.1, \mathrm{c}_{1}=-0.191837933, \mathrm{c}_{2}=-1.688934901, \mathrm{M}=0.3, \mathrm{k}_{1}=0.2, \mathrm{~m}=0.2, \mathrm{t}=5, \mathrm{k}_{2}=0.3\right\}$

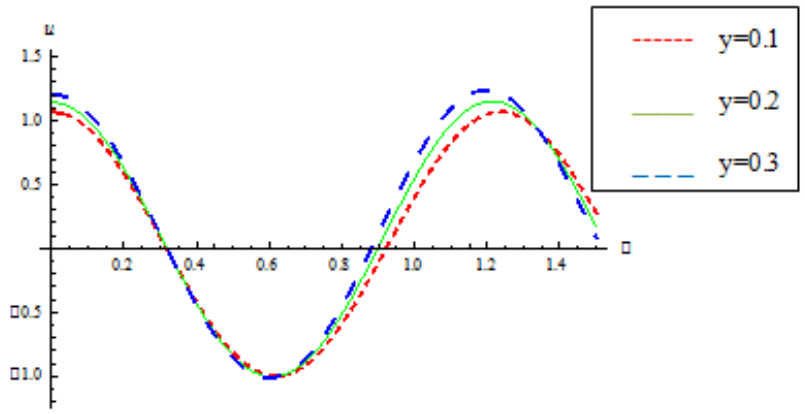

Fig.10:- the velocity u , Eq.( 59) for different value of y when keeping other parameters fixed $\left\{\delta=0.1, \mathrm{c}_{1}=-0.191837933, \mathrm{c}_{2}=-1.688934901, \mathrm{M}=0.3, \mathrm{k}_{1}=0.2, \mathrm{~m}=0.2, \mathrm{t}=5, \mathrm{k}_{2}=0.3, K=1\right\}$ 


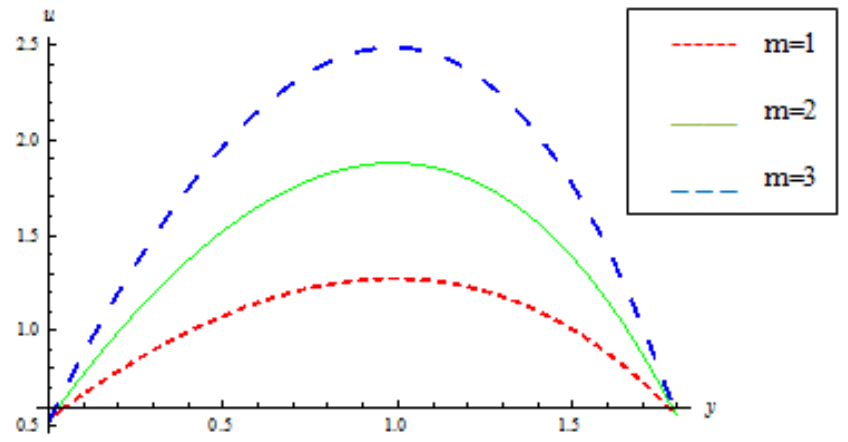

Fig.11:- the velocity u , Eq.( 59) for different value of $m$ when keeping other parameters fixed $\left\{\omega=0.2, \delta=0.1, \mathrm{c}_{1}=-0.191837933, \mathrm{c}_{2}=-1.688934901, \mathrm{k}_{1}=0.2, \mathrm{k}_{2}=0.3, \mathrm{M}=0.3, \mathrm{t}=5, K=1\right\}$

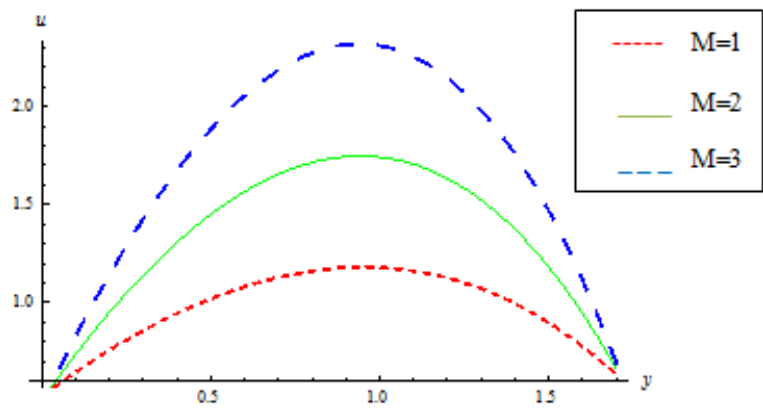

Fig.12:- the velocity u , Eq.( 59) for different value of $M$ when keeping other parameters fixed $\left\{\omega=0.2, \delta=0.1, \mathrm{c}_{1}=-0.191837933, \mathrm{c}_{2}=-1.688934901, \mathrm{k}_{1}=0.2, \mathrm{k}_{2}=0.3, \mathrm{~m}=0.2, \mathrm{t}=5, K=1\right\}$

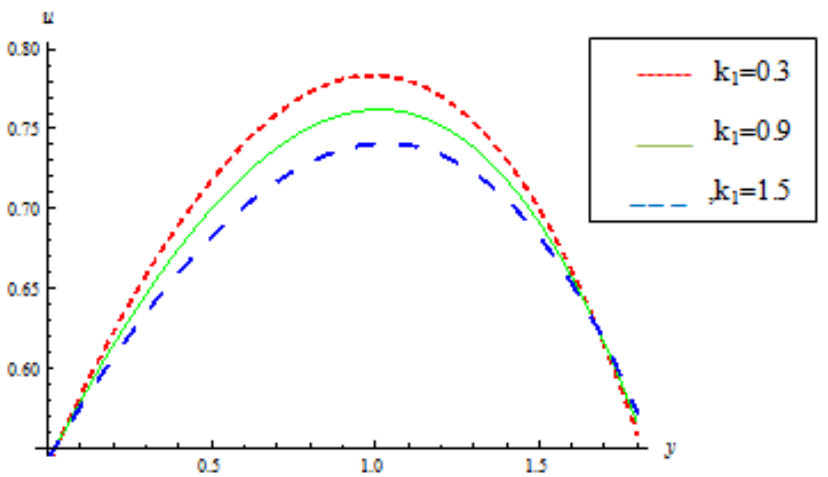

Fig.13:- the velocity u , Eq.( 59) for different value of $k_{1}$ when keeping other parameters fixed $\left\{\omega=0.2, \delta=0.1, \mathrm{c}_{1}=-0.191837933, \mathrm{c}_{2}=-1.688934901, \mathrm{M}=0.3, \mathrm{k}_{2}=0.3, \mathrm{~m}=0.2, \mathrm{t}=5, K=1\right\}$

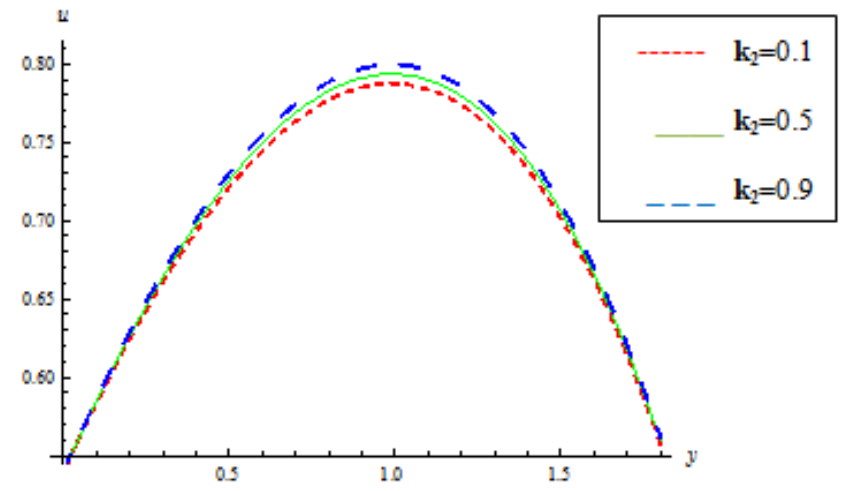

Fig.14:- the velocity u , Eq.( 59) for different value of $\mathrm{k}_{2}$ when keeping other parameters fixed $\left\{\omega=0.2, \delta=0.1, \mathrm{c}_{1}=-0.191837933, \mathrm{c}_{2}=-1.688934901, \mathrm{k}_{1}=0.2, \mathrm{~m}=0.2, \mathrm{t}=5, K=1\right\}$ 


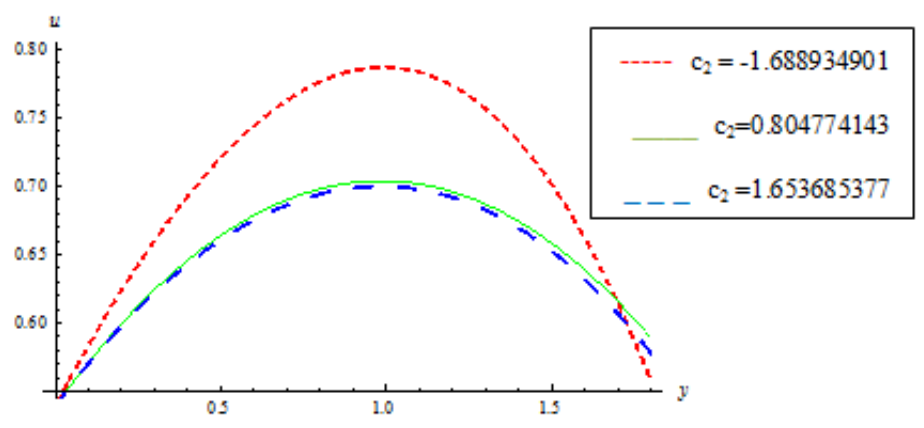

Fig.15:-the velocity u , Eq.( 59) for different value of $c_{2}$ when keeping other parameters fixed $\left\{\omega=0.2, \delta=0.1, \mathrm{c}_{1}=-0.191837933, \mathrm{M}=0.3, \mathrm{k}_{1}=0.2, \mathrm{~m}=0.2, \mathrm{k}_{2}=0.3, \mathrm{t}=5, K=1\right\}$

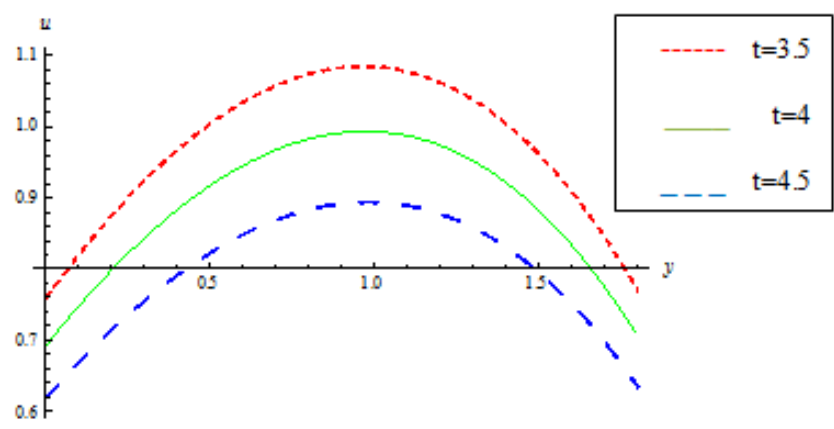

Fig.16:- the velocity u , Eq.( 59) for different value of $t$ when keeping other parameters fixed $\left\{\omega=0.2, \delta=0.1, \mathrm{c}_{1}=-0.191837933, \mathrm{c}_{2}=-1.688934901, \mathrm{M}=0.3, \mathrm{k}_{1}=0.2, \mathrm{~m}=0.2, \mathrm{k}_{2}=0.3, K=1\right\}$

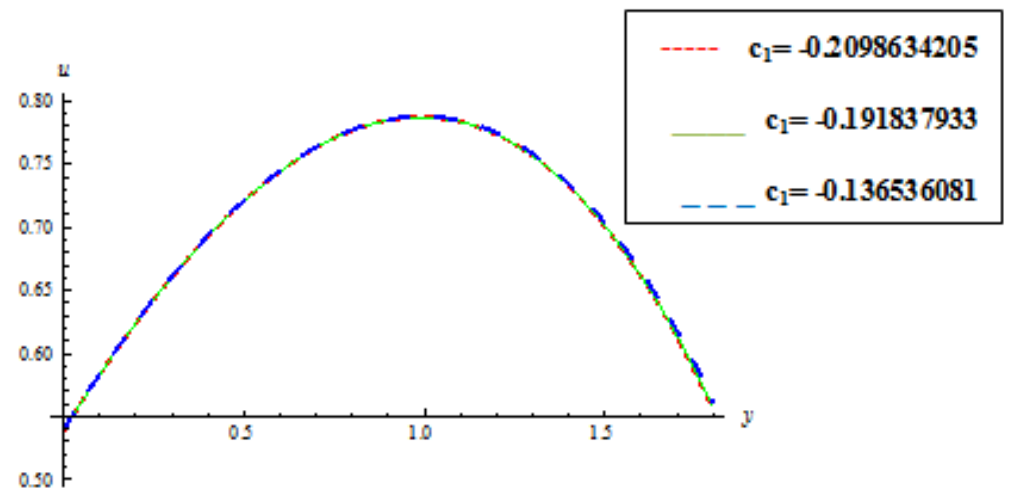

Fig.17:- the velocity u , Eq.( 59) for different value of $c_{1}$ when keeping other parameters fixed $\left\{\omega=0.2, \delta=0.1, \mathrm{c}_{2}=-1.688934901, \mathrm{M}=0.3, \mathrm{k}_{1}=0.2, \mathrm{~m}=0.2, \mathrm{t}=5, \mathrm{k}_{2}=0.3, K=1\right\}$

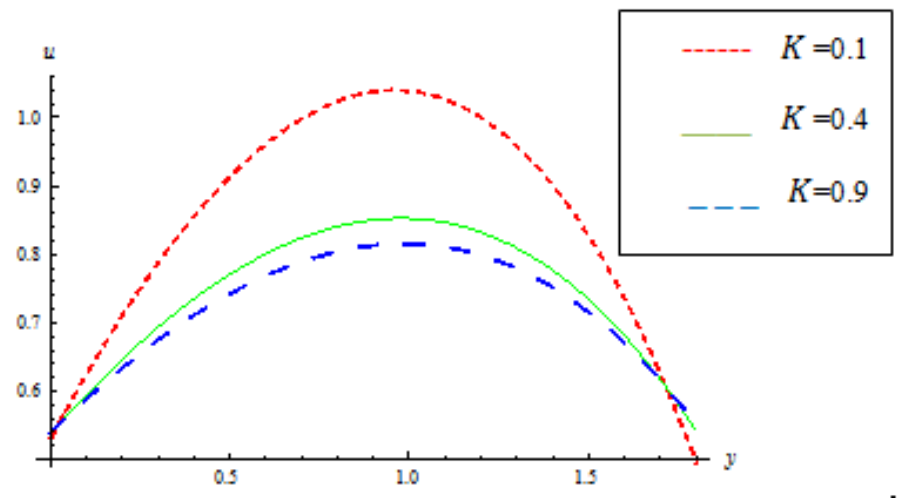

Fig.18:- the velocity u , Eq.( 59) for different value of $K$ when keeping other parameters fixed $\left\{\omega=0.2, \delta=0.1, \mathrm{c}_{1}=-0.191837933, \mathrm{c}_{2}=-1.688934901, \mathrm{M}=0.3, \mathrm{k}_{1}=0.2, \mathrm{~m}=0.2, \mathrm{t}=5, \quad \mathrm{k}_{2}=\mathbf{0 . 3}\right\}$ 


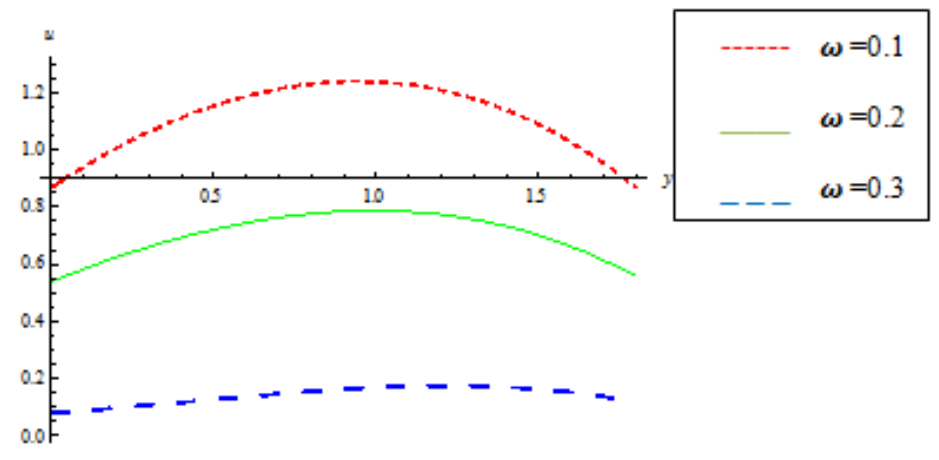

Fig.19:- the velocity u , Eq.( 59) for different value of $\omega$ when keeping other parameters fixed $\left\{\delta=0.1, \mathrm{c}_{1}=-0.191837933, \mathrm{c}_{2}=-1.688934901, \mathrm{M}=0.3, \mathrm{k}_{1}=0.2, \mathrm{~m}=0.2, \mathrm{t}=5, \mathrm{k}_{2}=0.3, K=1\right\}$

\section{References}

[1]. Anakira , NP. , Alomari AK. and Hashim . ; "Optimal homotopy asymptotic method for Solving Delay Differential Equations, ," Hindawi Pub Corp Math Problems in Eng. 1 (2013) 1-13

[2]. Burdujan , L. ; "The flow of a particular class of Oldroyd-B fluids” , J. Series on Math and its Applications. 3 (2011) $23-45$.

[3]. Fetecau , C. , Hayat T. and Fetecau C. ; " Starting solutions for oscillating motions of Oldroyd-B fluids in cylindrical domains" , Journal of Non-Newtonian fluid Mechanics. 153 (2008) 191-201.

[4]. Fetecau , C. , Sharat C. , Prasad SC. and Rajagapal , KRA. ; " A note on the flow iducad by a constantly accelerating plate in an Oldroyd-B fluid", Applied Mathematical Modeling . 31 (2007) 647-654.

[5]. Haitao , Q. and Mingyu , X. ; " Some unsteady unidirectional flows of a generalized Oldroyd-B fluid with fractional derivative " , Applied math .Modeling . 33 (2009) 4184-4191.

[6]. Kashkan , Bs. ; "Application of Optimal homotopy asymptotic method for the Approximate solution of Kawahara Equation" , . Applied Mathematical science .8 (2014) 875-884.

[7]. Lie , L. and Zhang, L. ; “Axial MHD flow of generalized Oldroyd-B fluid due to two oscillating cylinder ” , Advance material research .335 (2012) 83-86.

[8]. Mabood , F. and Ismail Al. , Ha , I. ; " Application of Optimal Homotopy asymptotic method for the Approximate solution of Riccat Equation ,“. Sains Malaysiana . 42 (2013) 863-867.

[9]. Mabood , F. and Khan ,WA . ; “ Approximate analytic solutions for influence of heat transfer on MHD stagnation point flow in porous medium ", .Computers\& Fluids. 100 (2014) 72-78.

[10]. Marinca , V., Herisam , N. , Bota , C. and Marinca , B. ; “An Optimal homotopy asymptotic method applied to the steady flow of a fourth grade fluid past a porous plate” , . Appl . Math .Lett. 22 (2009) 245-251.

[11]. Marinca V. and Herisanu , N. ; “ Optimal homotopy asymptotic approach to nonlinear oscillators with discontinuities " , Scientifi Research and Essays. 8 (2013) 161-167.

[12]. Shahid , N. , Rana, M . and Siddique ; “Exact solution for motion of an Oldroyd-B fluid over an infinite flat plate that applies an Oscillating shear stress to the fluid", Boundary Value Problems . 48 (2012) 1-19.

[13]. Shah , RA. , Islam , S. , Zeb , M . , and Ali , I .; " Optimal homotopy asymptotic method for thin film flows of flows of a third grade fluid", J of Advanced Research in Scientific Computing. 3 (2) (2011) 1-14.

[14]. Taza, G. , Saeed , Is ., and Rehan , A . ; “ Unsteady MHD Thin Film Flow of on Oldroyd-B fluid over on Oscillating Inclined Belt ", PLOS ONE . 10(7) (2015) 1-18. 